O'Dwyer, S.T., Moyle, W., Zimmer-Gembeck, M. \& De Leo, D. (2013). Suicidal ideation in family carers of people with dementia: A pilot study. International Journal of Geriatric Psychiatry, 28(11), 1182-1188.

\title{
Suicidal ideation in family carers of people with dementia: A pilot study
}

Running head: Suicidal ideation in dementia carers

Key words: Suicide, dementia, caregivers, mental health

Key points:

- Despite high rates of depression and hopelessness in family carers, there has been no research on suicidal ideation in this group

- In a sample of 120 family carers, $26 \%$ had contemplated suicide more than once in the past year

- $30 \%$ of suicidal carers indicated that they were likely to attempt suicide in the future

- More research is needed to confirm these findings and immediate action is required to identify and support suicidal carers in the community.

Authors: Siobhan T. O'Dwyer ${ }^{1,2}$, Wendy Moyle ${ }^{1,2}$, Melanie Zimmer-Gembeck ${ }^{2,3}$, Diego De Leo $^{4}$

Affiliations: ${ }^{1}$ Research Centre for Clinical and Community Practice Innovation, Griffith University; ${ }^{2}$ Griffith Health Institute; ${ }^{3}$ School of Applied Psychology, Griffith University; ${ }^{4}$ Australian Institute for Suicide Research and Prevention, Griffith University

Corresponding Author:

Dr Siobhan O'Dwyer

Griffith University

170 Kessels Rd

Nathan

QLD 4111

AUSTRALIA

P: 61737356619

F: 61737353560

E: s.odwyer@griffith.edu.au

Sponsors: Dr Siobhan O'Dwyer is supported by a Postdoctoral Research Fellowship from the Dementia Collaborative Research Centre (Carers and Consumers). The research was funded by a Griffith Health Institute Project Grant.

Word count: 3593

Abstract word count: 190 


\begin{abstract}
Objective:

The objective of this pilot study was to gather preliminary evidence on suicidal ideation in family carers of people with dementia
\end{abstract}

Methods:

An online, cross-sectional survey was conducted with 120 family carers, the majority of whom were located in Australia and the USA. The survey included measures of suicidality, self-efficacy, physical health, depression, hopelessness, anxiety, optimism, caregiver burden, coping strategies, and social support.

Results:

Twenty-six percent of carers had contemplated suicide more than once in the previous year. Only half of these had ever told someone they might commit suicide and $30 \%$ said they were likely to attempt suicide in the future. Carers who had contemplated suicide had poorer mental health, lower self-efficacy for community support service use, and greater use of dysfunctional coping strategies than those who had not. In a logistic regression, only depression predicted the presence of suicidal thoughts.

\title{
Conclusions:
}

A significant number of people might contemplate suicide while caring for a family member with dementia. While more research is required to confirm this finding, there are clear implications for policy and clinical practice in terms of identifying and supporting carers who are already contemplating suicide. 


\section{Introduction}

Around the world more than 35 million people have dementia and their families provide the "cornerstone of care" (Alzheimer's Disease International, 2009, p.54). In Australia, for example, the majority of community-dwelling people with dementia are cared for by a family member and $25 \%$ of these carers provide 40 hours of care or more per week (Access Economics, 2009). Further, one-third of that group continue caring at that level for five years or more (Access Economics, 2009).

As a result of the cognitive impairment and behavioural and psychological symptoms associated with dementia, caring for a person with dementia is acknowledged to be more burdensome than caring for a person with a physical disability, and it is well documented that the health and wellbeing of family carers can suffer as a result (Access Economics, 2009; Sorensen et al., 2006). In fact, as a group, carers are known to experience higher than normal rates of depression, anxiety and hopelessness (Cohen, 2000; Pinquart and Sorensen, 2003).

Depression, anxiety, and hopelessness have all been linked to suicidal ideation in the general population (Conwell and Thompson, 2008), but there have been no specific studies of suicidal ideation in family carers of people with dementia.

There has, however, been one study of suicidality in other informal caregivers. Rosengard and Folkman (1997) found that more than half of the spousal carers of gay men with AIDS in their study experienced suicidal ideation (irrespective of their own HIV status) and that these thoughts were associated with a perceived lack of social support, lack of social 
integration, and caregiver burden. Due to the social and political context of AIDS care in the mid-1990s, however, these findings may not translate to carers of people with dementia. The only other evidence of suicidality in carers comes from studies of homicide-suicides committed by older adults. In those studies, up to $50 \%$ of homicide-suicides were committed by spousal carers, with approximately $20 \%$ of those caring for a spouse with dementia (Cohen, 2000; Bourget et al., 2010). These studies, however, necessarily rely on information collected from medical reports and third parties after the event and provide only limited indication of suicidal thoughts during the caregiving journey. Furthermore, they have focused almost entirely on male carers (who are the most common perpetrators of homicide-suicide) (e.g. Malphurs and Cohen, 2005; Malphurs, Eisdorfer and Cohen, 2001), while the majority of dementia carers are older women (Alzheimer's Association, 2009; Alzheimer's Disease International, 2011).

The aims of this pilot study were to (1) collect preliminary evidence on rates of suicide ideation, (2) identify differences in physical health and psychosocial wellbeing between 'suicidal' and 'non-suicidal' carers, and (3) identify physical and psychosocial factors which predict suicidality in family carers of people with dementia.

\section{Methods}

\section{Recruitment}

Calls for participants were placed on six online carer discussion boards hosted by Australian, US and UK organisations and individuals in February 2012. Three of the websites were dementia-specific (including Alzheimer's and fronto-temporal dementia); one site hosted a number of different disease-specific forums, one of which was Alzheimer's Disease; and the 
remaining two sites were for carers at large, regardless of the care recipient's illness. The information invited carers to participate in a study on the physical and emotional wellbeing of carers. People were eligible to participate in the study if they could read English and identified themselves as the primary caregiver for a person with dementia who was still living in the community (i.e. not in long-term care). The survey was conducted online using LimeSurvey (LimeSurvey Project, Hamburg, Germany). To minimise participant burden, a 'save and return later' function was included in the survey. The study was approved by the University Human Research Ethics Committee. All participants were fully informed about the nature of the study prior to participation (including the presence of questions on suicidal ideation and suicide attempts) and completion of the survey was taken as consent. Where questions about suicide appeared, contact information was provided for telephone support or crisis lines in the relevant countries. A three-month timeframe was set for the collection of surveys, with all surveys submitted by end April 2012 included in the sample.

\section{Measures}

The selection of measures was guided by previous research on both caregiver burden and suicidality and was informed by the experiences of a Consumer Advisory Panel comprising peak bodies for caring and dementia in Australia, service providers, and former carers. The survey comprised the following self-report scales:

The Revised Memory and Behavior Problems Checklist (Teri et al., 1992) is a 24-item measure of behavioural problems in the person with dementia (PWD) and the caregiver's reaction to those problems. Two overall scores are created, where higher scores reflect, respectively, more frequent behaviours (RBMPC_Behavior) and more intense reactions 
(RBMPC_Reaction). The behaviour and reaction scales are reported to have reliabilities of .84 and .90 , respectively (Teri et al., 1992). In the current study Cronbach's alphas were .87 and 89 , respectively.

The Fortinsky Caregiver Self-efficacy Scale (Fortinsky et al., 2002) is a 10-item measure of self-efficacy. Nine of the items are used to create two subscales - self-efficacy for symptom management (FSE_Symptoms) and self-efficacy for community support service use (FSE_Community). Higher scores indicate greater self-efficacy. Internal consistency for the two scales has been reported as .77 and .78, respectively (Fortinsky et al., 2002). In the current study, Cronbach's alphas were .86 and .82 , respectively.

The Alzheimer's Disease Knowledge Scale (DKS; Carpenter et al., 2009) is 30-item test of knowledge about Alzheimer's disease risk factors, symptoms and management. It comprises 30 statements, each with a true/false response option. For the purposes of this study, the term 'Alzheimer's Disease' in each statement was replaced with 'dementia'. Correct responses are summed to create a total score, with higher scores indicating better knowledge. The reliability has been reported as .71 (Carpenter et al., 2009). In the current study, Cronbach's alpha was .51. Given the low alpha, the responses to each question were inspected. The proportion of participants answering correctly was more than $70 \%$ on all but four items (with the majority of items answered correctly by more than $90 \%$ of participants). The four items that were answered incorrectly by $30 \%$ of participants or more addressed life expectancy after diagnosis, blood pressure and cholesterol as risk factors, and the benefits of psychotherapy for depression in people with dementia. 
The SF-12 Health Survey Version 2 (Ware et al., 2005) is a 12-item generic measure of health. For the purposes of this analysis, the Physical Health Component Summary Scores (SF12_PCS) was created according to the recommended algorithm (Ware et al., 2005). The Mental Health Component was not calculated because individual measures of specific psychological constructs (e.g. depression, hopelessness) were included in the survey. Reliability estimates for the PCS have been reported as .89 (Ware et al., 2005) and in the current study Cronbach's alpha was .88.

The Center for Epidemiological Studies Depression Scale (CES-D; Radloff, 1977) is a 20-item measure of depressive symptomatology in the previous week. Higher scores indicate more depressive symptoms. Internal consistency has been reported as .85 (Radloff, 1977). In the current study, Cronbach's alpha was .91.

The Beck Hopelessness Scale (BHS; Beck et al., 1974) is a 20-item scale comprising statements about attitudes toward the future. Higher scores indicate greater levels of hopelessness. The internal consistency has been reported as .93 (Beck et al., 1974). In the current study, Cronbach's alpha was .93.

The Geriatric Anxiety Inventory (GAl; Pachana et al., 2007) is a20-item measure of anxiety symptoms in the past week. Higher scores indicate more symptoms of anxiety. The internal consistency has been reported as .91. In the current study, Cronabch's alph was .93.

The Life Orientation Test (LOT; Scheier and Carver, 1985) is a 12-item measure of dispositional optimism. Eight items contribute to the final score, where higher scores 
indicate greater optimism. Internal consistency has been reported as .76 (Scheier and Carver, 1985). In the current study, Cronbach's alpha was .92.

The Zarit Burden Scale (Zarit et al., 1985) is a 22-item measure of the sense of burden resulting from providing care. In the current study, the Cronbach's alpha was .90.

The Brief COPE (Carver 1997) is a 28-item measure of coping strategies. Guided by the work of Cooper et al. (2008), three subscales were created - emotion-focused, problem-focused, and dysfunctional coping strategies - with higher scores indicating greater use of the relevant strategies. Internal consistencies have been reported as $.72, .84$, and .75 , respectively (Cooper et al., 2008). In the current study, Cronbach's alpha was .72, .82, and .64 , respectively.

The Duke Social Support Index (Koenig et al., 1993) is a measure of social interactions and perceived support. The 11-item version, used in the Australian Longitudinal Study on Women's Health (Pachana et al., 2008) and comprising two subscales, was used. For the purposes of this analysis only the Satisfaction subscale (DSSI_Satisfaction) was used, with higher scores indicating a greater level of satisfaction with social support. Internal consistency has been reported as .80 . In the current study, Cronbach's alpha was .88 .

The Suicidal Behaviours Questionnaire-Revised (Osman et al., 2001) is a 4-item measure of past suicidal thoughts and behaviours and perceived likelihood of future suicidal behaviour. Item Two asks participants How often have you thought about killing yourself in the past year?, with response options of Never, Rarely (1 time), Sometimes (2 times), Often (3-4 
times), and Very often (5 or more times). For the purposes of this analysis, participants who responded Never or Rarely were classified as non-suicidal, while those who responded Sometimes, Often or Very Often were classified as suicidal. Item Three asks Have you ever told someone that you were going to commit suicide, or that you might do it?, with response options of No; Yes, at one time, but I did not really want to die; Yes, at one time, and I really wanted to die; Yes, more than once, but I did not want to do it; and Yes, more than once, and I really wanted to do it. Participants who selected any of the 'Yes' responses were classified as having disclosed their suicidal thoughts or intentions. Item Four asks How likely is it that you will attempt suicide someday? With response options of Never, No chance at all, Rather Unlikely, Unlikely, Likely, Rather Likely and Very Likely. Participants who responded Likely, Rather Likely or Very Likely were classified as 'Likely to Attempt' with the remainder classified as 'Unlikely to Attempt'.

A range of demographic and care-related information was also collected, including age, gender, relationship to person with dementia and length of time spent caring.

\section{Analysis}

The survey data were transferred from LimeSurvey to PASW Statistics Version 20.0 (SPSS Inc, Chicago, USA) for analysis. Basic frequencies and descriptive analyses were run on all scales and demographic data to inspect the distribution of responses and check for outliers. Timestamps were inspected to check the validity of the responses and surveys that had been completed in less than 30 minutes were inspected individually to check for implausible response patterns. There was no missing or invalid data on any of the main measures. There was a small amount of missing data for participants' dates of birth $(n=14)$. The format used 
for date-related questions in LimeSurvey is less than intuitive and, despite clear instructions, some participants had difficulty responding to this question. There was no indication that the format of any other questions in the survey was problematic. Cronbach's alpha was calculated to check the internal consistency of the scales. Descriptive analyses were conducted for participant demographics, the number of participants contemplating suicide, the number reporting suicidal intentions to others and the number likely to attempt suicide in the future. Independent t-tests were used to identify differences between suicidal and non-suicidal carers on the other scales. Where significant differences were identified, variables were entered as predictors into a logistic regression with suicidality ('suicidal' / 'non-suicidal') as the outcome variable. Analyses were considered significant at $p<0.05$.

\section{Results}

Sample

One hundred and twenty family carers completed the survey within the three-month timeframe. Participant demographics can be seen in Table 1. To summarise briefly, participants were aged between 25 and 80 years $(\underline{M}=59)$, with the majority from either Australia ( $n=51$ ) or the USA ( $n=56)$. Eighty-nine percent of carers were female and the majority were caring for a spouse or long-term partner.

Insert Table 1 here

Twenty-six percent $(n=32)$ had thought about suicide more than once in the previous 12 months and were classified as suicidal. Of these, only half $(n=16)$ had ever told someone 
they might commit suicide and approximately one-third $(n=9)$ were classified as likely to attempt suicide in the future.

Univariate analyses identified a number of significant differences between suicidal and nonsuicidal carers (see Table 2). Levene's Test for Equality of Variances was significant for SF12_PCS, GAI, and DSSI_Satisfaction, so adjusted $t$-statistics and degrees of freedom are reported for these variables. Suicidal carers reported more behavioural and psychological symptoms in the person with dementia $(p=0.048)$ and stronger reactions to these symptoms $(p=0.006)$. They also had lower self-efficacy for community support service use $(p=0.016)$, lower levels of optimism $(p=0.000)$, and were less satisfied with the social support they were receiving $(p=0.001)$. Suicidal carers reported higher levels of burden $(p=$ $.003)$, hopelessness $(p=0.000)$, depression $(p=0.000)$ and anxiety $(p=0.000)$. The use of dysfunctional coping strategies was greater among suicidal carers $(p=0.002)$, but there were no significant differences between suicidal and non-suicidal carers in the use of emotion-focused or problem-focused coping strategies. There were also no significant differences between groups for self-efficacy for symptom management, knowledge of dementia, or physical health.

Insert Table 2 here

Variables that were significantly different between groups in the univariate analyses were entered as predictors into a multivariate logistic regression, using forced entry, with suicidality (suicidal or non-suicidal) as the dependent variable. Collinearity diagnostics conducted on the predictor variables showed no variance inflation factors (VIF) above 10 or 
Tolerances below .1 (Field, 2005). The model was significant $\left(\chi^{2}(10)=45.39, p=0.000\right)$, but depression was the only significant individual predictor of suicidality ( $p=0.003$; see Table 3$)$. The results indicate that as depression scores increase so do the odds of contemplating suicide $(\exp b=1.12,95 \% \mathrm{Cl}=1.04,1.21$; see Table 3$)$.

Insert Table 3 here

\section{Discussion}

To our knowledge, this is the first survey study of suicidality in family carers of people with dementia and the findings indicate that 1 in 4 had contemplated suicide more than once in the past year. This is more than double the lifetime suicidal ideation rate reported for an Australian adult sample in a World Health Organization community survey (Bertolote et al., 2005) and more than eight times the annual adult rate of suicidal ideation recorded in a Centers for Disease Control survey of US adults in 2008-2009 (Centers for Disease Control and Prevention, 2011). It is also generally consistent with the findings of Rosengard and Folkman (1997), who reported that $12 \%$ of the AIDS carers in their study were classified as 'Higher Ideation' and 22\% were classified as 'Lower Ideation'.

Only half of the carers who were classified as suicidal had ever told someone they might die by suicide. While this could be interpreted to mean that the thoughts were not accompanied by a clear plan or a strong intention to act, nearly $30 \%$ of suicidal carers reported that they were likely to attempt suicide in the future. It may be that the potential implications of revealing these thoughts (such as stigma, hospitalisation, or having the person with dementia removed from their care) prevented carers from disclosing them. 
While this could not be determined from the current data, it is consistent with anecdotal evidence from carers involved in our research. It is also consistent with the concept of 'hidden ideators' - those who are suicidal and potentially at risk of attempting, but do not disclose their thoughts or plans unless directly asked - that has been reported in the suicide literature more generally (Morrison and Downey, 2000). While suicidal thoughts will not necessarily lead to a suicide attempt, the importance of specifically asking family carers about suicidal thoughts (and providing appropriate support if such thoughts are present) is a clear implication for both research and practice (Morrison and Downey, 2000). In fact, given that the majority of family carers of people with dementia are aged over 55 years and that up to $75 \%$ of people over 50 who die by suicide have never made a previous attempt (Alzheimer's Association, 2011; Conwell \& Thompson, 2008), this should be considered a matter of some urgency.

There were significant differences between 'suicidal' and 'non-suicidal' carers on a range of psychosocial outcomes. In particular, carers who had contemplated suicide had poorer mental health, stronger reactions to the behavioural and psychological symptoms of dementia, and more negative perceptions of the social support they were receiving. They also had lower self-efficacy for making use of community support services and were engaging in more dysfunctional coping strategies. While the direction of the relationship cannot be determined from a cross-sectional study (e.g. do dysfunctional coping strategies lead to suicidal ideation, or does the experience of suicidal thoughts lead to the use of dysfunctional coping strategies?), these findings are largely consistent with previous research on suicide risk more generally (Conwell and Thompson, 2008; McLean et al., 2008; Van Orden and Conwell, 2011) and highlight some of the specific factors that might be 
associated with suicide risk in family carers of people with dementia. While risk factors do not generate suicidal thoughts, the identification of a set of specific risk factors for suicidal ideation in family carers will be important if screening tools or targeted suicide prevention programs are to be developed for this population.

Despite significant univariate differences between suicidal and non-suicidal carers on almost all of the psychosocial factors measured in the current study, in a multivariate model only depression significantly predicted suicidality. As depression is acknowledged to be a very important risk factor for suicide (Conwell et al., 2011; McLean et al., 2008), this is not surprising. The lack of other significant predictors in the model, however, was unexpected and could indicate that depression is mediating the relationship between suicidal ideation and factors such as reactions to dementia symptoms, satisfaction with social support, and dysfunctional coping strategies. While an exploration of mediation was beyond the scope of this study, mediating relationships of this nature have been suggested in the suicide literature more generally (e.g. Conwell and Thompson, 2008) and should be explored in future research on suicidality in dementia carers. The possibility of interactions between predictor variables should also be considered.

This study has a number of potential limitations. These include the small sample size, the use of an online survey, and the recruitment of carers from online discussion boards. In the absence of previous studies on the rate of suicidal ideation in this population, it was impossible to calculate a target sample size. Even with the small sample, however, an alarming number of carers reported contemplating suicide and clear differences between groups were evident. The use of an online survey limits the generalizability of the findings to 
carers without internet access. Similarly, generalizability is also limited by the recruitment of carers from online discussion boards who, it could be assumed, are already engaged in helpseeking behaviour and receiving social support (albeit in an online community). It could be argued, however, that carers who have financial restrictions or are socially isolated may be more likely to experience suicidal thoughts (Conwell et al., 2011; McLean et al., 2008; Van

Orden and Conwell, 2011) and, as such, the current findings could actually be an underestimation of the rate of suicidal ideation in family carers. The use of multiple recruitment methods in future research could confirm the rates and improve generalizability. The study also included only those caring in the community, but carers who have placed the person with dementia into long-term care, or been bereaved, may also contemplate suicide. In their study of AIDS carers, Rosengard and Folkman (1997) found that when compared with the other classification groupings, significantly more of the carers classified as 'higher ideation' were bereaved. While it was not within the scope of this study to include those carers, it is a direction for future research. It was also not within the scope of this study to identify differences between US and Australian carers or carers with different demographic profiles, but in a larger study these could be explored. Finally, while this study focused on family carers of people with dementia, future research should also focus on the experiences of family members caring for people with other long-term illnesses or disabilities, who may also contemplate suicide.

\section{Conclusion}

This pilot study suggests that an alarming number of people contemplate suicide while caring for a family member with dementia. While a larger study is required to confirm the rate of suicidal ideation in this population, it is clear that some family carers are already 
contemplating suicide and, as such, health professionals and community care providers must work to identify and support these carers now. The presence of depression may provide an indication of risk, but there is also an imperative for those in clinical and community practice to directly assess the presence of suicidal thoughts. With half the suicidal carers never having disclosed their thoughts, and one-third indicating that they might attempt suicide in the future, there is a real and urgent need for further research and practical recommendations in this area.

\section{Acknowledgements}

Dr Siobhan O'Dwyer is supported by a Postdoctoral Research Fellowship from the Dementia Collaborative Research Centre (Carers and Consumers). This research was funded by a Griffith Health Institute Project Grant. The authors wish to thank the owners and moderators of The Alzheimer's Spouse, Dementia Cafe, Healing Well, Carers.org, FTD Support Forum and Carers Haven; the carers who generously gave up their time to complete the survey; Sarah Walbank (Carers Queensland) and the Consumer Advisory Panel for their assistance in the development of the survey instrument; and Billy Sung for technical assistance and preliminary data analysis.

\section{References}

Access Economics. 2009. Making choices: Future dementia care - projects, problems and preferences. Alzheimer's Australia: Canberra. 
Alzheimer's Association. 2011. Alzheimer's Association Report: 2011 Alzheimer's disease facts and figures. Alzheimers Dement 7: 208-244.

Alzheimer's Disease International. 2009. World Alzheimer Report 2009. Alzheimer's Disease International: London.

Beck AT, Weissman A, Lester D, Trexler L. 1974. The measurement of pessimism: The Hopelessness Scale. J Consult Clin Psych 42: 861-863.

Bertolote JM, Fleischmann A, De Leo D et al. 2005. Suicide attempts, plans, and ideation in culturally diverse sites: the WHO SUPRE-MISS community survey. Psychol Med 35: 14571465.

Bourget D, Gagne P, Whitehurst L. 2010. Domestic homicide and homicide-suicide: The older offender. J Am Acad Psychiatry 38: 305-311.

Carpenter BD, Balsis S, Otilingam PG, Hanson PK, Gatz, M. 2009. The Alzheimer's Disease Knowledge Scale: Development and psychometric properties. Gerontologist 49: 236-247.

Carver CS. 1997. You want to measure coping but your protocol's too long: Consider the Brief COPE. Int J Beh Med 4: 92-100.

Centres for Disease Control and Prevention. Suicidal thoughts and behaviours among adults aged $\geq 18$ years - United States, 2008-2009. MMWR 2011; 60 (No. SS-13). 
Cohen D. 2000. Caregivers for persons with Alzheimer's disease. Curr Psychiatry

Rep 2: 32-39.

Conwell Y, Thompson C. 2008. Suicidal behaviour in elders. Psychiat Clin N Am 31: 333-56.

Conwell Y, Van Orden K, Caine ED. 2011. Suicide in older adults. Psychiat Clin N Am 34: 451468.

Field A. 2005. Discovering statistics using SPSS ( ${ }^{\text {nd }}$ Ed). SAGE Publications: London.

Fortinsky RH, Kercher K, Burant CJ. 2002. Measurement and correlates of family caregiver self-efficacy for managing dementia. Aging Ment Health 6: 153-160.

Koenig HG, Westlund RE, George LK et al. 1993. Abbreviating the Duke Social Support Index for use in chronically ill elderly individuals. Psychosomatics 34: 61-69.

Malphurs JE, Cohen D. 2005. A statewide case-control study of spousal homicide-suicide in older persons. Am J Geriatr Psychiatry 13: 211-217.

Malphurs JE, Eisdorfer C, Cohen, D. 2001. A comparison of antecedents of homicide-suicide and suicide in older married men. Am J Geriatr Psychiatry 9: 49-57. 
McLean J, Maxwell M, Platt S, Harris F, Jepson R. 2008. Risk and protective factors for suicide and suicidal behaviour: A literature review. Scottish Government Social Research: Scotland.

Morrison LL, Downey DL. 2000. Racial differences in self-disclosure of suicidal ideation and reasons for living: Implications for training. Cultur Divers Ethnic Minor Psychol 6: 374-386.

Osman A, Bagge CL, Gutierrez PA et al. 2001. The Suicidal Behaviors Questionnaire-Revised (SBQ-R): Validation with clinical and nonclinical samples. Assessment 8: 443-454.

Pachana NA, Byrne GJ, Siddle H et al. 2007. Development and validation of the Geriatric Anxiety Inventory. Int Psychogeriatr 19: 103-114.

Pachana NA, Smith N, Watson M, McLaughlin D, Dobson A. 2008. Responsiveness of the Duke Social Support sub-scales in older women. Age Ageing 37: 666-672.

Pinquart M, Sorensen S. 2006. Helping caregivers of persons with dementia: Which interventions work and how large are their effects? Int Psychogeriatr 18: 577-595.

Radloff LS. 1977. The CES-D Scale: A self-report depression scale for research in the general population. Appl Psych Meas 1: 385-401.

Rosengard C, Folkman S. 1997. Suicidal ideation, bereavement, HIV serostatus and psychosocial variables in partners of men with AIDS. AIDS Care 9: 373-84. 
Scheier MF, Carver CS. 1985. Optimism, coping and health: Assessment and implications of generalized outcome expectancies. Health Psychol 4: 219-247.

Sorensen S, Duberstein P, Gill D, Pinquart M. 2006. Dementia care: Mental health effects, intervention strategies, and clinical implications. Lancet Neurol 5: 961-973.

Teri L, Truax P, Logsdon R et al. 1992. Assessment of behavioural problems in dementia: The Revised Memory and Behavior Problems Checklist. Psychol Ageing 7: 622-631.

Van Orden K, Conwell Y. 2011. Suicides in late life. Curr Psychiatr Rep 13: 234-241.

Ware JE, Kosinski M, Turner-Bowker DM, Gandek B. 2005. How to Score Version 2 of the SF12 Health Survey. QualityMetric Incorporated: Rhode Island.

Zarit SH, Orr NK, Zarit, JM. 1985. The hidden victims of Alzheimer's Disease: Families under stress. New York University Press: New York. 
Table 1. Participant demographics

\begin{tabular}{|c|c|c|}
\hline Variable & $M(S D)$ & $\mathbf{N}$ \\
\hline Age & $\begin{array}{c}58.76 \\
(10.67)\end{array}$ & \\
\hline Time spent caring (years) & $\begin{array}{c}5.14 \\
(3.54)\end{array}$ & \\
\hline \multicolumn{3}{|l|}{ Gender } \\
\hline Male & & 13 \\
\hline Female & & 107 \\
\hline \multicolumn{3}{|l|}{ Country of Residence } \\
\hline Australia & & 51 \\
\hline Canada & & 1 \\
\hline USA & & 56 \\
\hline UK & & 9 \\
\hline Other & & 3 \\
\hline \multicolumn{3}{|l|}{ Relationship to PWD ${ }^{1}$} \\
\hline Spouse & & 82 \\
\hline Son & & 2 \\
\hline Daughter & & 30 \\
\hline Son-in-Law & & 1 \\
\hline Daughter-in-Law & & 4 \\
\hline Other & & 1 \\
\hline \multicolumn{3}{|l|}{ Dementia Type ${ }^{2}$} \\
\hline Unsure / No formal diagnosis & & 16 \\
\hline Alzheimer's Disease & & 67 \\
\hline Fronto Temporal Dementia & & 18 \\
\hline Dementia with Lewy Bodies & & 3 \\
\hline Vascular Dementia & & 15 \\
\hline Other & & 12 \\
\hline
\end{tabular}

${ }^{1} \mathrm{PWD}=$ Person with Dementia

${ }^{2}$ This question allowed participants to choose all options that applied 
Table 2. Differences between 'suicidal' and 'non-suicidal' carers on measures of health and psychosocial wellbeing.

\begin{tabular}{|c|c|c|c|c|c|}
\hline Measure & $\begin{array}{c}\text { Non- } \\
\text { Suicidal } \\
\text { (n= 88) } \\
M(S D)\end{array}$ & $\begin{array}{l}\text { Suicidal } \\
\text { (n= 32) } \\
M(S D)\end{array}$ & $\begin{array}{c}t \\
\text { (df) }\end{array}$ & $\mathbf{p}$ & $95 \% \mathrm{Cl}^{*}$ \\
\hline \multirow[t]{2}{*}{ RMBPC_Behavior } & 63.78 & 70.06 & -2.00 & .048 & $-12.50,-0.06$ \\
\hline & $(15.12)$ & $(15.50)$ & $(118)$ & & \\
\hline \multirow[t]{2}{*}{ RMBPC_Reaction } & 52.92 & 63.75 & -2.81 & .006 & $-18.46,-3.20$ \\
\hline & $(18.64)$ & $(18.70)$ & $(118)$ & & \\
\hline \multirow[t]{2}{*}{ FSE_Symptoms } & 27.97 & 26.22 & .74 & .459 & $-2.91,6.40$ \\
\hline & (11.13) & $(12.10)$ & $(118)$ & & \\
\hline \multirow[t]{2}{*}{ FSE_Community } & 21.35 & 16.47 & 2.44 & .016 & $0.92,8.84$ \\
\hline & (9.31) & $(10.68)$ & $(118)$ & & \\
\hline \multirow[t]{2}{*}{ DKS } & 22.14 & 22.22 & -0.18 & .858 & $-0.99,0.83$ \\
\hline & $(2.28)$ & $(2.08)$ & $(118)$ & & \\
\hline \multirow[t]{2}{*}{ SF12_PCS } & 50.89 & 47.57 & 1.87 & .068 & $-0.26,6.90$ \\
\hline & (7.37) & (9.04) & $(46.82)^{\dagger}$ & & \\
\hline \multirow[t]{2}{*}{ CES-D } & 18.90 & 33.88 & -7.07 & .000 & -19.17, - \\
\hline & $(10.42)$ & (9.79) & (118) & & 10.78 \\
\hline \multirow[t]{2}{*}{ BHS } & 7.28 & 12.91 & -4.81 & .000 & $-7.94,-3.31$ \\
\hline & (5.61) & (5.79) & $(118)$ & & \\
\hline \multirow[t]{2}{*}{ GAI } & 7.49 & 13.41 & -6.12 & .000 & $-7.84,-3.99$ \\
\hline & (6.10) & $(4.05)$ & $(83.17)^{\dagger}$ & & \\
\hline \multirow[t]{2}{*}{ LOT } & 18.97 & 13.06 & 4.20 & .000 & $3.12,8.69$ \\
\hline & (6.93) & $(6.44)$ & $(118)$ & & \\
\hline \multirow[t]{2}{*}{ Zarit Burden } & 48.57 & 57.16 & -3.03 & .003 & $-14.20,-2.98$ \\
\hline & $(14.02)$ & $(12.84)$ & $(118)$ & & \\
\hline \multirow[t]{2}{*}{ Brief COPE_Emotion-focused } & 14.39 & 12.91 & 1.35 & .180 & $-0.70,3.66$ \\
\hline & (5.19) & (5.67) & $(118)$ & & \\
\hline \multirow[t]{2}{*}{ Brief COPE_Problem-focused } & 10.18 & 9.59 & .69 & .489 & $-1.09,2.27$ \\
\hline & $(4.20)$ & (3.83) & $(118)$ & & \\
\hline \multirow[t]{2}{*}{ Brief COPE_Dysfunctional } & 8.28 & 11.28 & -3.21 & .002 & $-4.85,-1.15$ \\
\hline & $(4.27)$ & $(5.16)$ & $(118)$ & & \\
\hline \multirow[t]{2}{*}{ DSSI_Satisfaction } & 15.38 & 12.94 & 3.41 & .001 & $1.00,3.88$ \\
\hline & $(2.73)$ & $(3.69)$ & $(43.96)^{\dagger}$ & & \\
\hline
\end{tabular}

*Confidence Interval of the Difference +Equal variances not assumed 
Table 3. Outcomes of a logistic regression predicting suicidality.

\begin{tabular}{|c|c|c|c|c|c|}
\hline \multirow[t]{2}{*}{ Predictors } & \multirow[t]{2}{*}{$\beta$ (SE) } & \multirow[t]{2}{*}{ df } & \multirow[t]{2}{*}{$\exp b$} & \multicolumn{2}{|c|}{ 95\% Cl for Exp B } \\
\hline & & & & Lower & Upper \\
\hline RMBPC_Behaviour & $\begin{array}{l}-0.01 \\
(0.02)\end{array}$ & 1 & 0.99 & .94 & 1.04 \\
\hline RMBPC_Reaction & $\begin{array}{c}0.01 \\
(0.19)\end{array}$ & 1 & 1.01 & .97 & 1.05 \\
\hline FSE_Community & $\begin{array}{l}-0.08 \\
(0.03)\end{array}$ & 1 & 0.99 & .93 & 1.05 \\
\hline CESD & $\begin{array}{c}0.12 \\
(0.04)\end{array}$ & 1 & 1.12 & 1.04 & 1.21 \\
\hline BHS & $\begin{array}{c}0.09 \\
(0.07)\end{array}$ & 1 & 1.09 & .95 & 1.26 \\
\hline GAI & $\begin{array}{c}0.06 \\
(0.07)\end{array}$ & 1 & 1.06 & .92 & 1.22 \\
\hline LOT & $\begin{array}{c}0.07 \\
(0.07)\end{array}$ & 1 & 1.07 & .93 & 1.22 \\
\hline Zarit Burden & $\begin{array}{c}-0.01 \\
(0.03)\end{array}$ & 1 & 0.99 & .93 & 1.05 \\
\hline Brief COPE_Dysfunctional & $\begin{array}{c}0.05 \\
(0.06)\end{array}$ & 1 & 1.05 & .93 & 1.19 \\
\hline DSSI_Satisfaction & $\begin{array}{l}-0.04 \\
(0.10)\end{array}$ & 1 & 0.96 & .79 & 1.17 \\
\hline Constant & $\begin{array}{l}-5.64 \\
(2.98)\end{array}$ & 1 & 0.004 & & \\
\hline
\end{tabular}

